\title{
Single parameter FPT-algorithms for non-trivial games
}

\author{
Vladimir Estivill-Castro and Mahdi Parsa \\ School of ICT, Griffith University, Queensland, Australia 4111 \\ \{v.estivill-castro,m.parsa\}@griffith.edu.au.com
}

\begin{abstract}
We know that $k$-UNIFOrm NASH is $W[2]$-Complete when we consider imitation symmetric win-lose games (with $k$ as the parameter) even when we have two players. However, this paper provides positive results regarding Nash equilibria. We show that consideration of sparse games or limitations of the support result in fixed-parameter algorithms with respect to one parameter only for the $k$-UNIFORM NASH problem. That is, we show that a sample uniform Nash equilibrium in $r$-sparse imitation symmetric win-lose games is not as hard because it can be found in FPT time (i.e polynomial in the size of the game, but maybe exponential in $r$ ). Moreover, we show that, although NP-Complete, the problem of Best NASH EQUILIBRIUM is also fix-parameter tractable.
\end{abstract}

Key words: Algorithmic Game Theory, Computational Complexity.

\section{Introduction}

Game theory analyzes interactions between self-interested agents, with the recent interest in artificial intelligence, multi-agents systems, and automatic decision making it has received much study. The first complexity results for computing Nash equilibria used classic notions of complexity theory [11]. Later, several researchers have introduced different types of equilibria and games. These NP-hardness results have been extended to the other games and solution concepts $[1,2,5,6]$.

We study the fixed-parameter tractability of NP-Hard problems for the computation of Nash equilibria. One of the most recently studied class of games are win-lose games $[2,5]$. In these games, all payoff values are 0 or 1 . We study the parameterized complexity of finding uniform Nash equilibria in imitation win-lose games because:

- The computation complexity of a Nash equilibrium in win-lose games is as hard as for general bi-matrix games [1].

- There is a corresponding one-to-one relation between Nash equilibria of two-player games and Nash strategies for the row player in an imitation game [5].

- "A uniform mixed strategy is the simplest way of mixing pure strategies", but deciding the existence of uniform Nash equilibria in win-lose games is NP-Complete [2] and it is $W[2]$-Hard [8] in bi-matrix games.

- Deciding whether an imitation symmetric win-lose game has a uniform Nash equilibrium with support of size $k$ is $W[2]$-Complete [7]. 
- It has been observed [12] that the lower bounds of Chen et al [3] and the $W[2]$-hardness results imply that unless $\mathrm{FPT}=W[1]$, there is no $n^{o(k)}$ time algorithm for computing a Nash equilibrium with support size at most $k$ in a bi-matrix game.

In contrast, if the support is known, the equilibrium can be found in polynomial time. Thus, there is much interest in studying the complexity of the support size or if the support is included in a set of strategies. We show that restrictions of the support result in fixed-parameter algorithms.

\section{$2 \quad$ FPT results for win-lose games}

A win-lose game $\mathcal{G}=(A, B)$ is called $r$-sparse if there are at most $r$ nonzero entries in each row and each column of the matrices $A$ and $B$. The first natural step to parameterize the computation of a sample Nash equilibrium is to consider the $r$ as a parameter in $r$-sparse games. But, Chen, Deng and Teng [4] showed that it is unlikely to find an $\epsilon$-approximate equilibrium for a 10-sparse game in time polynomial both in $\epsilon$ and $n$ (the size of game). Therefore, it is unlikely to find an FPT-time algorithm that just considers $r$ as the parameter. We have proved the parameterized tractability of Nash equilibria in a subclass of $r$-sparse games.

Definition 1 Let $\boldsymbol{I S W L G}$ be the class of all Imitation Symmetric WinLose Games $\left(I_{n \times n}, M_{n \times n}\right)$ where the matrix $M$ is a symmetric matrix, and has diagonal equal to zero.

If a game $(I, M)$ is in ISWLG, then this game represents a simple undirected graph $G=(V, E)$ where the matrix $M$ corresponds with $G$ 's adjacency matrix. We have shown that any maximal clique in the graph representation of game $\mathcal{G}=(I, M)$ corresponds to a uniform Nash equilibrium, but the reverse is not true.

Lemma 2 Let $G$ be the graph representation of game $\mathcal{G}=(I, M)$ in $\boldsymbol{I S} \boldsymbol{W L} \boldsymbol{G}$ and $G_{\boldsymbol{x}}$ be a maximal clique of size $k$ of $G$. Then the mixed strategy profile $(\boldsymbol{x}, \boldsymbol{x})$ constitutes a uniform Nash equilibrium of $\mathcal{G}$ where $\boldsymbol{x}$ is defined as: $x_{i}=\left\{\begin{array}{l}1 / k, \text { if } i \text { is vertex of } G_{\boldsymbol{x}} ; \\ 0, \quad \text { otherwise. }\end{array}\right.$

We study the effect of sparsity. Existence of uniform Nash equilibria is not an issue since every graph has a maximal clique. By Lemma 2, every game in ISWLG has a uniform Nash equilibrium.

Theorem 3 Finding a uniform Nash equilibrium for a $r$-sparse game in $\boldsymbol{I S} \boldsymbol{W L} \boldsymbol{G}$ is polynomial in the size of the game but exponential in $r$.

We used the link between graph theory and Nash equilibria to show our FPT results. Now, we can provide many results regarding families of 
graphs where finding a maximal clique is in FPT. For example we show that a sample uniform Nash equilibrium can be found in FPT-time where the treewidth of graph is considered as the parameter.

Theorem 4 Let $\mathcal{G}=(I, M)$ be an imitation symmetric win-lose game with graph representation $G$. If $G$ has bounded tree-width $\omega$, then a uniform Nash equilibrium of the $\mathcal{G}$ can be found in $O\left(2^{\omega} \cdot \omega \cdot|I|\right)$ time.

\section{FPT results when searching Nash equilibrium on a set}

We can obtain a result for general bi-matrix games. The following problem has been shown to be NP-Complete.

NASh Equilibrium In A SubSeT

Instance : A game $\mathcal{G}=(A, B)$.

Parameter: A subset of strategies $E_{i} \subseteq\{1, \ldots, n\}$ for each player $i$.

Question : Does there exists a Nash equilibrium of $\mathcal{G}$ in which all strategies not included in $E_{i}$ are played with probability zero?

There is a Feasibility Program [13], which is a linear program, and, if the support of a Nash equilibrium is known, then the computation of corresponding Nash equilibrium can be done in polynomial time. We use this Feasibility Program to proof following theorem.

Theorem 5 Nash Equilibrium In A Subset is in FPT.

\section{FPT results for congestion games}

In congestion games (also routing games), players choose several links, one link to route their traffic [10, and references].

Definition 6 A routing game $\mathcal{G}$ consists of:

- a set of $m$ parallel links from a source node $s$ to a terminal node $t$ and a capacity $c^{j}$ for each link $j \in\{1,2, \ldots, m\}$, - a set $N=\{1,2, \ldots, n\}$, of $n$ users,

- traffic weights, $w_{1}, w_{2}, \ldots, w_{n}$, where the $i$-th user has traffic $w_{i}>0$.

A pure strategy for a user $i$ is a link $j$ in $\{1,2, \ldots, m\}$. Analogously, a pure strategy profile is an $n$-tuples $\left(l_{1}, l_{2}, \ldots l_{n}\right)$, when user $i$ chooses link $l_{i}$ in $\{1,2, \ldots, m\}$. The cost for a user $i$, when users choose a pure strategy profile $P=\left(l_{1}, l_{2}, \ldots, l_{n}\right)$ is $C_{i}(P)=\sum_{k: l_{k}=l_{i}} w_{k} / c^{l_{i}}$. Every routing game admits at least one pure Nash equilibrium [9]. However, the individual (non-cooperative) optimization of utility does not lead to a social optimal outcome. Therefore, the price of stability is a measure inefficiency of equilibria. It differentiates between games that all Nash equilibria are inefficient or some of them are inefficient. Formally, the price of stability of a game is the ratio between the best objective function value of a 
Nash equilibrium of the game and the optimal outcome. We consider the makespan as the social objective function. The makespan of a strategy profile $P=\left(l_{1}, l_{2}, \ldots, l_{n}\right)$ is defined as: $C_{\max }(P)=\max _{i \in\{1,2, \ldots, n\}} C_{i}(P)$.

\section{Best Nash Equilibrium}

Instance : A routing game $\mathcal{G}$ with identical links.

Parameter $: k \in \mathbb{N}$.

Question : Is there a pure Nash equilibrium $P$ with $C_{\max }(P) \leq k$ ?

Best Nash Equilibrium on identical links is a NP-Hard problem [9], but we showed it is fix parameter tractable. with a parameterized reduction to Integer Linear Programming. The Integer Linear ProGRAMMING problem (with a number of variables bounded by the parameter) is FPT.

Theorem 7 Best NAsh Equilibrium is in FPT.

\section{References}

1. T. Abbott, D. Kane, and P. Valiant. On the complexity of two-player win-lose games. FOCS '05 p 113-122. IEEE Computer Soc.

2. V. Bonifaci, U. D. Iorio, and L. Laura. The complexity of uniform Nash equilibria and related regular subgraph problems. Theoretical Computer Science, 401(13):144-152, 2008.

3. J. Chen, B. Chor, M. Fellows, X. Huang, D. Juedes, I.A. Kanj, and X. Xia. Tight lower bounds for certain parameterized NP-hard problems. Information and Computation, 201(2):216 - 231, 2005.

4. X. Chen, X. Deng, and S. H. Shang-Hua Teng. Sparse games are hard. WINE 2006, vol. 4286 LNCS, p 262-273. Springer.

5. B. Codenotti and D. Stefankovic. On the computational complexity of Nash equilibria for $(0,1)$ bimatrix games. Information Processing Letters, 94:145-150, 2005.

6. V. Conitzer and T. Sandholm. Complexity results about Nash equilibria. IJCAI03,, p 765-771, Acapulco, Mexico, Morgan Kaufmann.

7. V. Estivill-Castro and M. Parsa. The parameterized complexity of uniform Nash equilibria in win-lose games. Manuscript submitted for publication.

8. V. Estivill-Castro and M. Parsa. Computing Nash equilibria gets harder- new results show hardness even for parameterized complexity. The Australasian Theory Symposium (CATS2009), vol 94 of CRPIT, Australian Computer Soc.

9. D. Fotakis, S. Kontogiannis, E. Koutsoupias, M. Mavronicolas, and P. Spirakis. The structure and complexity of Nash equilibria for a selfish routing game. Theoretical Computer Science, 410(36):3305-3326, 2009.

10. E. Gassner, J. Hatzl, S.O. Krumke, H. Sperber, and G.J. Woeginger. How hard is it to find extreme Nash equilibria in network congestion games? Theoretical Computer Science, 410(47-49):4989-4999, 2009.

11. I. Gilboa and E. Zemel. Nash and correlated equilibria: Some complexity considerations. Games and Economic Behavior, 1(1):80-93, March 1989.

12. D. Hermelin, C. C. Huang, S. Kratsch, and Wahlsrtöm M. Parameterized twoplayer Nash equilibrium. personal communication.

13. B. von Stengel. Computing equilibria for two-person games. Handbook of Game Theory, vol 3, p 1723-1759, Amsterdam, 2002. Elsevier. Chapter 45. 